\title{
AN EMBEDDED FINGER VEIN RECOGNITION SYSTEM
}

\author{
Dary Ram.T.R. ${ }^{1}$ \\ ${ }^{I} P G$ Student,Department of ECE,MET'S School of Engineering,Kerala, India
}

\begin{abstract}
In this paper, an embedded finger-vein recognition system (FVRS) for authentication on ATM network is proposed. The system is implemented on an embedded platform and equipped with a novel finger-vein recognition algorithm. The proposed system consists of four hardware modules: radio frequency identification system, image acquisition module, embedded main board, and human machine communication module. RFID module will start the very initial communication between the user and the device. The finger-vein images of the user are acquired by the image acquisition module of the hardware. The finger-vein recognition algorithm is coded and targeted as firmware of the embedded main board. Human machine interface module communicates with the user through keyboard and displays the outcome of the match in human recognizable formats like LCD or LED displays as well as voice message. The RFID module enhances the security and credibility of the whole system by giving short message service (SMS) through GSM technology platform to the actual user.
\end{abstract}

Keywords: Embedded finger vein recognition system, RFID module, Embedded platform, communication module, image acquisition module etc

\section{INTRODUCTION}

In this hi-tech world number of crimes such as ATM robberies, unauthorized entering of people into large industries, unauthorized entering of data etc. are increasing day by day. The main causes of these problems are due to the defects in existing security systems. Digital security has acquired special importance due to vast amount of digital information and the high value that is frequently been attached to it. Normally we use passwords for security. Effective user authentication applications are crucial to protect information security. In response to the growing number of threats to data security, a wide variety of authentication mechanisms have been developed. Here we introduce a new security system which uses finger vein recognition system for authentication in ATM networks. Section 2 details the existing systems in security and its limitations. Section 3 deals with the proposed algorithm. Section 4 deals with proposed design which is explained along with working, its advantages, and applications and so on. Section 5 includes the experimental results.

\section{EXISTING SYSTEMS}

Existing authentication systems have evolved over several decades. Each system has been very carefully scrutinized by security experts as well as the end users in terms of the level of security provided by the system as well as the inconvenience caused to the end user. Personal Identification Number [PIN] based authentication system[1] is found to have major flaws like complete breach of security once the PIN is revealed as well as the agony to the end user to memorize the PIN. Biometrics came as the potent solution to this problem of PIN based system. Biometric patterns [2] like iris, face, palm/fingerprints, voice etc have been developed to attempt the for ultimate security that cannot be breached.

Notwithstanding the increasing complexity of implementation of the biometric based authentication system, no biometric system has yet been developed that provides a reasonably good convenience to the end user but at the same time provides impeccable defense against security breach. For example, iris patterns are unique and are medically proven to be stable after a year or so after birth. But it suffers from the inconvenience to the end user especially those using contact lenses as well as elderly people. Fingerprints and palm prints based authentication systems suffer from being frayed and do not ensure that specimen being authenticated is from a live body. Face recognition system pose difficulty when the person has different expression or face-lift [3] from that in the prestored data. Hence it has been a demanding task for authentication system developers to set a high threshold of identity validation that is impregnable to the ever adapting deceptive practices [4]. A successful authentication system that provides this security at the same time which provides fairly good convenience to end users will have several wide ranging application from consumer electronics, banking, airport, business establishments to space and defense segments.

\section{PROPOSED ALGORITHM}

The proposed algorithm has four steps, namely, image acquisition, image segmentation and alignment, image enhancement and feature extraction. The finger-vein images of users have to be acquired and stored as database. The 
algorithm is implementable in embedded hardware based platforms like PIC micro-controllers.

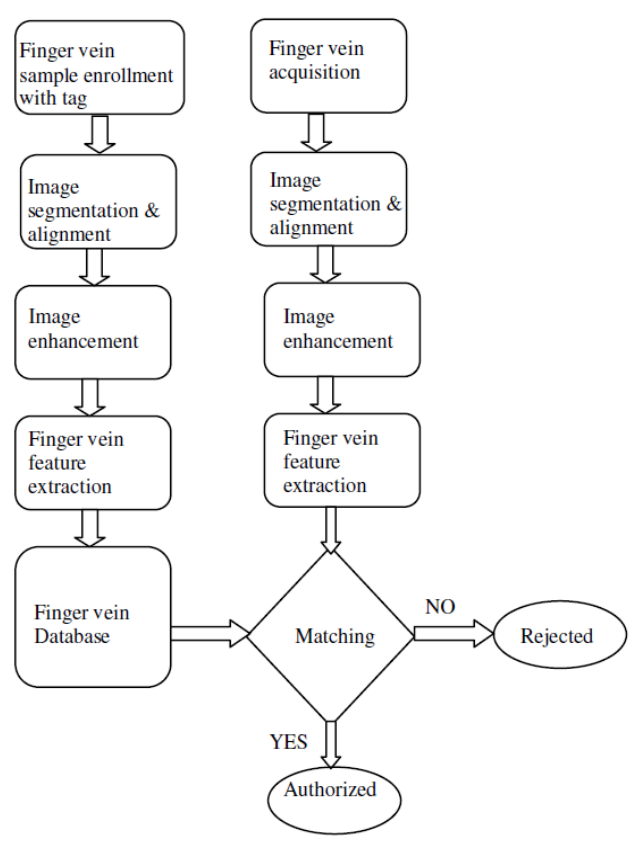

Fig.1.The flow-chart of the proposed finger vein recognition algorithm

The finger vein images of the user to be authenticated also goes through the same steps as during database collection stage. Authentication is done based on the feature match of the newly acquired finger-vein data with any of those in the prestored finger-vein database. The steps involved in the proposed algorithm are explained in the coming sections.

\subsection{Image Acquisition}

To obtain high quality near-infrared (NIR) images, a special device was developed for acquiring the images of the finger vein without being affected by ambient temperature. Generally, finger-vein patterns can be imaged based on the principles of light reflection[6] or light transmission .The device mainly includes the following modules: a Monochromatic camera of resolution $580 \times 600$ pixels, daylight cut-off filters (lights with the wavelength less than $800 \mathrm{~nm}$ are cut off), transparent acryl (thickness is $10 \mathrm{~mm}$ ), and the NIR light source. The transparent acryl serves as the platform for locating the finger and removing uneven illumination. The NIR light irradiates the backside of the finger. In , a light-emitting diode (LED) was used as the illumination source for NIR light. With the LED illumination source, however, the shadow of the finger-vein obviously appears in the captured images.

\subsection{Image Segmentation and Alignment}

Because the position of fingers usually varies across different finger-vein images, it is necessary to normalize the images before feature extraction and matching. The bone in the finger joint is articular cartilage. Unlike other bones, it can be easily penetrated by NIR light. When a finger is irradiated by the uniform NIR light, the image of the joint is brighter than that of other parts. Therefore, in the horizontal projection of a finger-vein image, the peaks of the projection curve correspond to the approximate position of the joints. Since the second joint of the finger is thicker than the first joint, the peak value at the second joint is less prominent. Hence, the position of the first joint is used for determining the position of the finger.

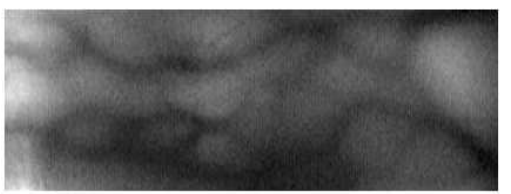

Fig.2. The ROI of the finger-vein image.

The alignment module includes the following steps. First, the part between the two joints in the finger-vein image is segmented based on the peak values of the horizontal projection of the image.

Second, a canny operator with locally adaptive threshold is used to get the single pixel edge of the finger. Third, the midpoints of finger edge are determined by edge tracing so that the midline can be obtained. Fourth, the image is rotated to adjust the midline of the finger horizontally. Finally, the ROI of the finger-vein image is segmented according to the midline

\section{3.Image Enhancement}

The segmented finger-vein image is then enhanced to improve its contrast.The image is resized to $1 / 4$ of the original size, and enlarged back to its original size. Next, the image is resized to $1 / 3$ of the original size for recognition. Bicubic interpolation is used in this resizing procedure. Finally, histogram equalization is used for enhancing the gray level contrast of the image.

\subsection{Feature Extraction}

The fractal model developed by Mandelbrot [7] provides an excellent method for representing the ruggedness of natural surfaces and it has serve data successful image analysis tool for image compression and classification. Since different fractal sets with obviously different textures may share the same fractal dimension[8] Fractal Dimension allows us to measure the degree of complexity by evaluating how fast our measurements increase or decrease as our scale becomes 
larger or smaller. Two types of fractal dimension: selfsimilarity dimension and box-counting dimension .Essentially, data behave with a power law relationship if they fit the following equation:

$$
\mathrm{y}=\mathrm{c}^{*} \mathrm{x}^{\wedge} \mathrm{d} \text { where } \mathrm{c} \text { is a constant. }
$$

One way to determine if data fit a power law relationship is to plot the $\log (\mathrm{y})$ versus the $\log (\mathrm{x})$. If the plot is a straight line, then it is a power law relationship.

\section{PROPOSED DESIGN}

Finger vein recognition (FVR) is very effective when compared pin number based authentication and other types of Biometric security methods like finger print security, palm print security, image scanning and some recognition techniques. Finger-vein being hidden inside the human body, is difficult to be duplicated. Also, it is very convenient for the end user due to non-intrusive nature of acquisition. FVR system uses the vein scanning. As it is related to the biological factor, it is very difficult to change the vein information of a user. So, this system can provide more security than any other security level. In this FVR system, we are focusing on high security with RFID technology. Initially each and every user will be given with one RFID secret card. This will make an effective initial communication between the user and the device. This technique will make the device to extract the user information from its memory. The transmitter section of the FVR system is shown in Figure 3 and the receiver section in Figure 4.

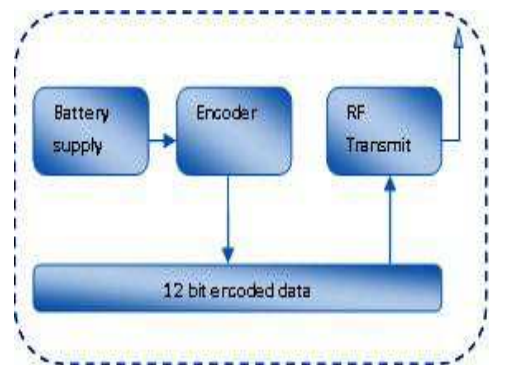

Fig 3: FVRS - transmitter section

In FVR system, the RFID module is used to collect the user database. With this system, a unique code will be generated for each and every user for storing the finger vein details in the server. Here an active RFID technology is used for creating the secret signal. The encoded signal will be continuously transmitted by the card if it is in on state. This RFID will reduce the complexity of the image acquisition module. As the RFID have an unique signal it can store only one vein information. So authentication and identification will become soon. Because of these features the FVRS will be a faster recognition system.

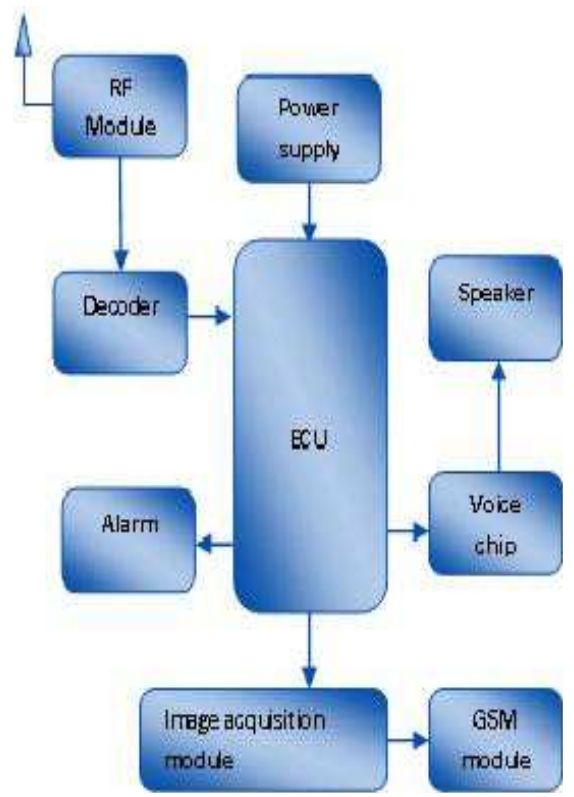

Fig 4: FVRS -receiver section

In this section, RF receiver module is attached with the embedded control unit. This unit receives the secret digital data from the card and it will be given to the controller. In the FVRS recognition unit, vein images will be stored in the image acquisition module. If RF receiver receives any digital code, then automatically code verification will be done inside the embedded control unit. If the code is matched then an asynchronous command will be given to the image acquisition module. Then the vein image comparison will be done inside the processor. If the image is matched then automatically the device will go to its working state.

To this ECU further a GSM module is interfaced. With this module a password system can be developed. During every successful access, automatically intimation will be given to the controlling authority. The unit will send a password with this intimation. It will make a very effective security to the user. This password will be working for one time. It will play an effective authentication process. This mobile GSM communication module will not only send the intimation for authorize but also for unauthorized.

\section{EXPERIMENTAL RESULTS}

Step by step experimental results of proposed Finger-vein recognition system is shown below. Demonstration of the proposed system is done by targeting the algorithm to a PIC 16F877A micro-controller based hardware. Image processing and Graphical User Interface (GUI) is developed in Matlab. The experimental demonstration is done using a five step process as explained below: 
Step1: Selection of pre stored finger vein sample

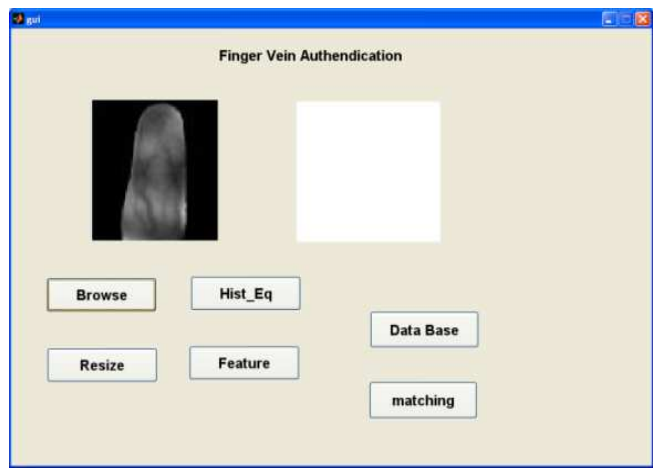

Step2: Image enhancement

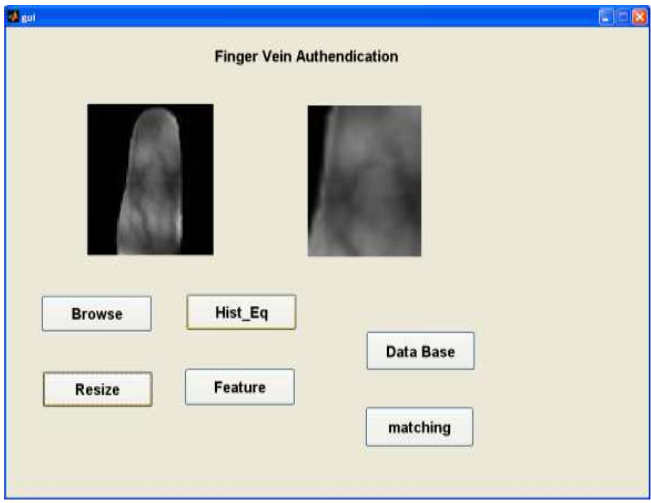

Step 3:Histogram equalization

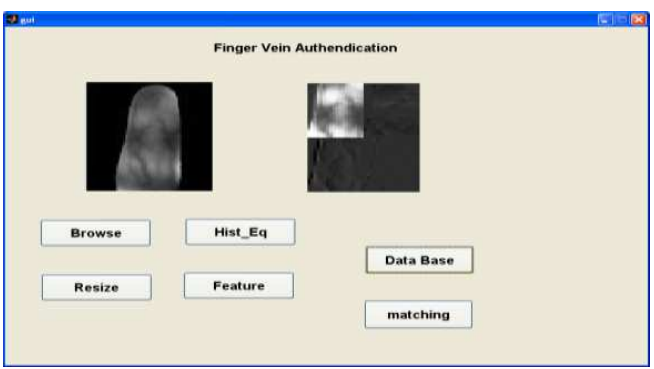

Step 4: Feature extraction

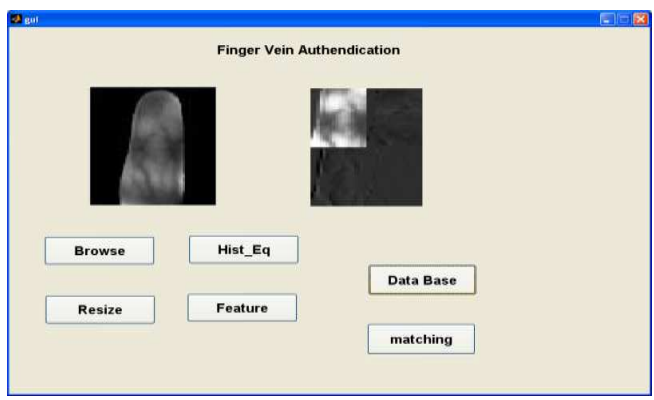

Step5: Matching

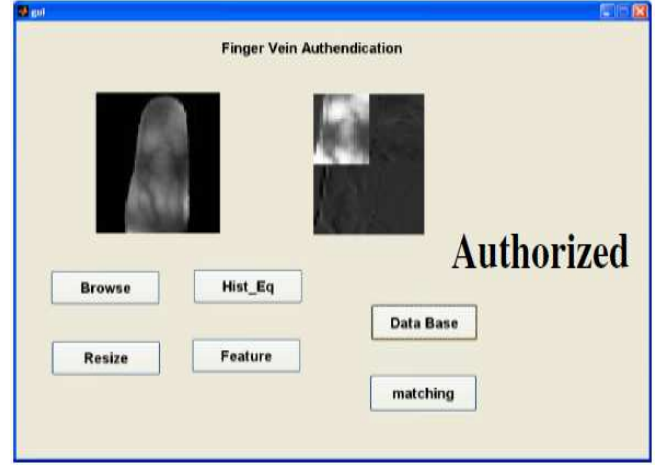

\section{COMPARISON WITH PREVIOUS METHODS}

A comparative evaluation of the major biometric technologies is described in Table 1. Each method has its own advantages and disadvantages. For example, fingerprint is the least expensive method and is commonly used for identifying suspected criminals. However, the age and occupation of a person may cause some difficulties in capturing a complete and accurate fingerprint image.

Table 1 comparison with previous methods

\begin{tabular}{l|l|l|l|l} 
Biometrics & Accuracy & Cost & $\begin{array}{l}\text { Security } \\
\text { Level }\end{array}$ & Stability \\
\hline Fingerprint & Medium & Low & Low & Low \\
\hline $\begin{array}{l}\text { Facial } \\
\text { Recognition }\end{array}$ & Low & High & Low & Low \\
\hline $\begin{array}{l}\text { Iris Scan } \\
\begin{array}{l}\text { Speaker } \\
\text { Recognition }\end{array}\end{array}$ & High & High & Medium & Medium \\
\hline $\begin{array}{l}\text { Hand } \\
\text { Geometry }\end{array}$ & Low & High & Low & Low
\end{tabular}

Fingerprints are also relatively easy to be copied, so that usage may be restricted when used for security purposes.

\section{CONCLUSIONS}

In this paper, a new finger-vein based user identification system is developed which overcomes the drawbacks of existing authentication systems. The proposed system provides excellent immunity against deceptive practices and is also very convenient to the users. Experimental results show high accuracy for correct match and rejection of wrong specimens. The proposed system will work efficiently in hardware as well as embedded platforms as is evident from the performance are PIC 16F877A based demonstration model. The proposed system can be effectively used for authentication in banking, consumer electronics, airport, space and defense applications. 


\section{REFERENCES}

[1]. A. K. Jain, S. Pankanti, S. Prabhakar, H. Lin, and A. Ross, "Biometrics: a grand challenge", Proceedings of the 17th International Conference on Pattern Recognition (ICPR), vol. 2, pp. 935-942, 2004.

[2]. P. Corcoran and A. Cucos, "Techniques for securing multimedia content in consumer electronic appliances using biometric signatures," IEEE Transactions on Consumer Electronics, vol 51, no. 2, pp. 545-551, May 2005.

[3]. Y. Kim, J. Yoo, and K. Choi, "A motion and similaritybased fake detection method for biometric face recognition systems," IEEE Transactions on Consumer Electronics, vol.57, no.2, pp.756-762, May 2011.

[4]. D. Wang , J. Li, and G. Memik, "User identification based on fingervein patterns for consumer electronics devices", IEEE Transactions on Consumer Electronics, vol. 56, no. 2, pp. 799-804, 2010.

[5]. H. Lee, S. Lee, T. Kim, and Hyokyung Bahn, "Secure user identification for consumer electronics devices," IEEE Transactions on Consumer Electronics, vol.54, no.4, pp.17981802, Nov. 2008.

[6]. Y. G. Dai and B. N. Huang, "A method for capturing the finger-vein image using non uniform intensity infrared light", Image and Signal Processing, vol.4, pp.27-30, 2008.

[7]. B. B. Mandelbrot, Fractals: Form, Chance and Dimension, San Francisco, CA: Freeman, 1977.

[8]. B.B.Mandelbrot and D.stuffer"antipodal correlations and the texture in critical percolation clusters "journal of physics:mathematical and general” vol. 27, pp. 237-242,

\section{BIOGRAPHIE}

DARY RAM.T.R. received the bachelor of Engineering degree in Electronics and Communication from S.N.M.I.M.T., Suld Maliankara, Ernakulam, Kerala, India in 2009.
Currently pursuing Master of Engineering degree from the department of VLSI Design, at MET'S School of Engineering, Mala, Thrissur, Kerala, India. 